\title{
Synthesis and Characterization of a Poly(ethylene glycol) Prepolymer To Be Applied as a Bioadhesive
}

\author{
P. Ferreira, ${ }^{1,2}$ J. F. J. Coelho, ${ }^{1}$ R. Pereira, ${ }^{1}$ António F. M. Silva, ${ }^{1}$ M. H. Gil ${ }^{1}$ \\ ${ }^{1}$ Departamento de Engenharia Química, Polo II, Universidade de Coimbra, 3030-790 Coimbra, Portugal \\ ${ }^{2}$ Escola Superior de Ciência e Tecnologia, Universidade Católica Portuguesa, Centro Regional das Beiras, \\ Estrada da Circunvalação, 3504-505, Viseu, Portugal
}

Received 23 May 2006; accepted 23 December 2006

DOI 10.1002/app.26206

Published online 27 March 2007 in Wiley InterScience (www.interscience.wiley.com).

\begin{abstract}
Surgical adhesives can be applied to wound closure and to covering and protecting surface wounds. Depending on their degradability, they can then slough off or can be reabsorbed by the organism when the wound initiates the healing process. In this work, we intended to develop a new urethane-based bioadhesive that could accomplish these purposes. Urethanes are considered to be promising candidates as adhesives because of the possibility of their synthesis as prepolymers and therefore their reaction with amino groups present in the biological molecules. Some urethanes were obtained by the reaction of poly(ethylene glycol) with isophorone diisocyanate. The characterization of the various materials was accomplished with differ-
\end{abstract}

ent techniques: attenuated total reflection/Fourier transform infrared, swelling-capacity determination, the evaluation of the moisture-curing kinetics, a reaction with aminated substrates (as a simulation of the living tissues), and the determination of the surface energy by contact-angle measurements. The study of the thermal properties of the urethanes was performed by dynamical mechanical thermal analysis, differential scanning calorimetry, and thermogravimetric analysis. The hemocompatibility of the urethane was also evaluated by thrombosis and hemolysis tests. (c) 2007 Wiley Periodicals, Inc. J Appl Polym Sci 105: 593-601, 2007

Key words: adhesion; swelling; thermal properties

\section{INTRODUCTION}

During the last decades, some revolutionary advances in the development of wound-closure methods, such as automatic suture devices (suture threads and staples), surgical strips, and even some biological glues, have been accomplished. However, both surgical suturing and stapling have been associated with several disadvantages, such as wound infection and sinus and granuloma formation in the case of resorbable sutures. ${ }^{1}$ For that reason, renewed interest has being growing among both surgeons and scientists for the development of new adhesives that could accomplish several tasks, such as the promotion of hemostasis and sealing air leakages.

There are several advantages in the use of an adhesive compared with conventional sutures or stapling: tissue bonding can be achieved rapidly, the need to remove sutures is eliminated, and the application of the adhesive is significantly less painful than the alternative processes.

Correspondence to: P. Ferreira (paula_calvinho@yahoo. com).

Contract grant sponsor: Fundação para a Ciência e Tecnologia (to P. F.); contract grant number: SFRH/BD/1052/ 2000.

Journal of Applied Polymer Science, Vol. 105, 593-601 (2007)

C 2007 Wiley Periodicals, Inc.

\section{(\$)WILEY}

InterScience
The most used surgical glues nowadays are fibrinbased adhesives ${ }^{2-4}$ and cyanoacrylates..$^{5-7}$ The fibrinbased adhesives present several problems, such as immunogenicity and risk of blood transmission diseases such as human immunodeficiency virus and bovine spongiform encephalopathy (BSE). On the other hand, cyanoacrylates have been reported to degrade in aqueous media to produce formaldehyde, which causes inflammation and has carcinogenicity potential. Other options are now coming to light, such as gelatin $^{8,9}$ and albumin-based glues. ${ }^{10}$ Among the synthetic materials, urethane-based adhesives have been considered to be the most promising. ${ }^{11}$ One of the main advantages of urethanes is their biocompatibility. Also, the presence of some free isocyanate groups in their structure allows them to react with the amino groups of the proteins existing in the living tissues. This reaction results in the formation of urea linkages and consequently the promotion of adhesion. An adhesive based on polyurethanes was synthesized and studied by Lipatova. ${ }^{11}$ Lipatova showed that the degradation products did not present any toxic effects and that the glue was autosterile and ensured intensive hemostasis. Its potential as a surgical adhesive was tested in several fields, such as renal surgery, ${ }^{12}$ endocrinology, ${ }^{13}$ and pancreatic occlusion. ${ }^{14}$

This article describes the modification of poly(ethylene glycol) (PEG) to obtain a prepolymer with terminal isocyanate groups. The characterization of the ma- 
terial was accomplished by different techniques: attenuated total reflection/Fourier transform infrared (ATR-FTIR), swelling-capacity determination, the study of the moisture-curing kinetics, a reaction with aminated substrates, and the determination of the surface energy by contact-angle measurements. The hemocompatibility of the urethane was also evaluated by thrombosis and hemolysis tests.

\section{EXPERIMENTAL}

\section{Materials}

All the reagents were purchased from Sigma-Aldrich Chemical Co. (Barcelona, Spain) and used with no further treatment. Venous rabbit blood, used in the hemocompatibility studies, was collected in polypropylene tubes with a $9 / 1 \mathrm{blood} /$ acid citrate dextrose (ACD) solution ${ }^{15}$ ratio and was used immediately after collection. The human plasma was gently supplied by the Imunohemoterapia Service at Coimbra University Hospital and was used after being frozen by this service. The blood type from which it was extracted was A positive.

\section{Synthesis}

Urethanes based on PEG 400 were synthesized by the modification of their hydroxyl groups with isophorone diisocyanate (IPD). The ratio of NCO groups to $\mathrm{OH}$ groups was $2 / 1$.

The reactions were performed by the stirring of the two components at $60^{\circ} \mathrm{C}$ under a nitrogen atmosphere. By the ATR-FTIR technique, it was possible to observe that after $24 \mathrm{~h}$ of reaction, all the PEG hydroxyl groups had reacted with the NCO groups of the isocyanate, resulting in the formation of urethane groups. This technique was also employed to confirm the presence of free isocyanate groups in the urethane. All the ATR-FTIR analyses were performed on a Magma-IR 750 spectrometer from Nicolet Instrument Corp., which was equipped with a Golden Gate single-reflection diamond ATR instrument. Spectra were recorded from an average of 128 scans at a resolution of $4 \mathrm{~cm}^{-1}$.

\section{Swelling-capacity evaluation and moisture-curing kinetics}

To determine the swelling capacity, three samples of the urethanes were primarily dried until a constant weight at $60^{\circ} \mathrm{C}$ under vacuum conditions. The weight of the dried sample was obtained $\left(W_{d}\right)$. These samples were then placed in a container with a saturated solution of pentahydrated copper sulfate and were weighted at different times until a constant weight was achieved $\left(W_{s}\right)$. The swelling ratio was evaluated with eq. (1):

$$
\text { Swelling ratio }(\%)=\left(\frac{W_{s}-W_{d}}{W_{d}}\right) \times 100
$$

The moisture-curing kinetics was determined by the evaluation of the stability of the isocyanate free groups present in the prepolymers when the samples were contained in a $100 \%$ water saturated atmosphere. This parameter was evaluated by the measurement of the height of the band at $2260 \mathrm{~cm}^{-1}$ by the ATR-FTIR technique, which corresponded to the NCO free groups, and the experiment was conducted until this band was no longer detected.

\section{Reaction with aminated substrates}

One of the advantages of the prepolymers is their reaction with the amino groups of the proteins existing in the living tissues. This reaction results in the formation of urea linkages, which result in the adhesion of the tissues involved. Figure 1 presents this reaction for the prepolymers synthesized during this work.

To evaluate the adhesive strength of the prepolymers, they were placed between gelatin sheets that contained a great number of amino groups and therefore could simulate the living tissues. The gelatin sheets were then subjected to a binding strength test using a Chatillon TCD 1000. The pulling velocity was $20 \mathrm{~mm} / \mathrm{min}$, and the assays were carried out at room temperature until the fracture of the gelatin sheets. The software program coupled to the apparatus registered the force and length variation.

\section{Determination of the surface energy by contact-angle measurements}

Surface tensions of the urethanes and gelatin sheets were evaluated by static contact-angle measurements in an OCA 20 from Dataphysics. The tests were performed on the air-facing surfaces of the samples with four liquids-water, formamide, ethylene glycol, and propylene glycol-with the sessile drop method. Nine measurements on different points were performed to calculate the mean static contact angle and its standard deviation. According to the OwensWendt-Rabel and Kaelble relationships, the dispersive and polar components of the urethanes as well as those of the gelatin sheets were determined.

\section{Thermal properties}

Thick specimens $\left(15.20 \times 7.45 \times 1.10 \mathrm{~mm}^{3}\right)$ were analyzed by dynamical mechanical thermal analysis (DMTA). A Triton Tritec 2000 analyzer was used in 

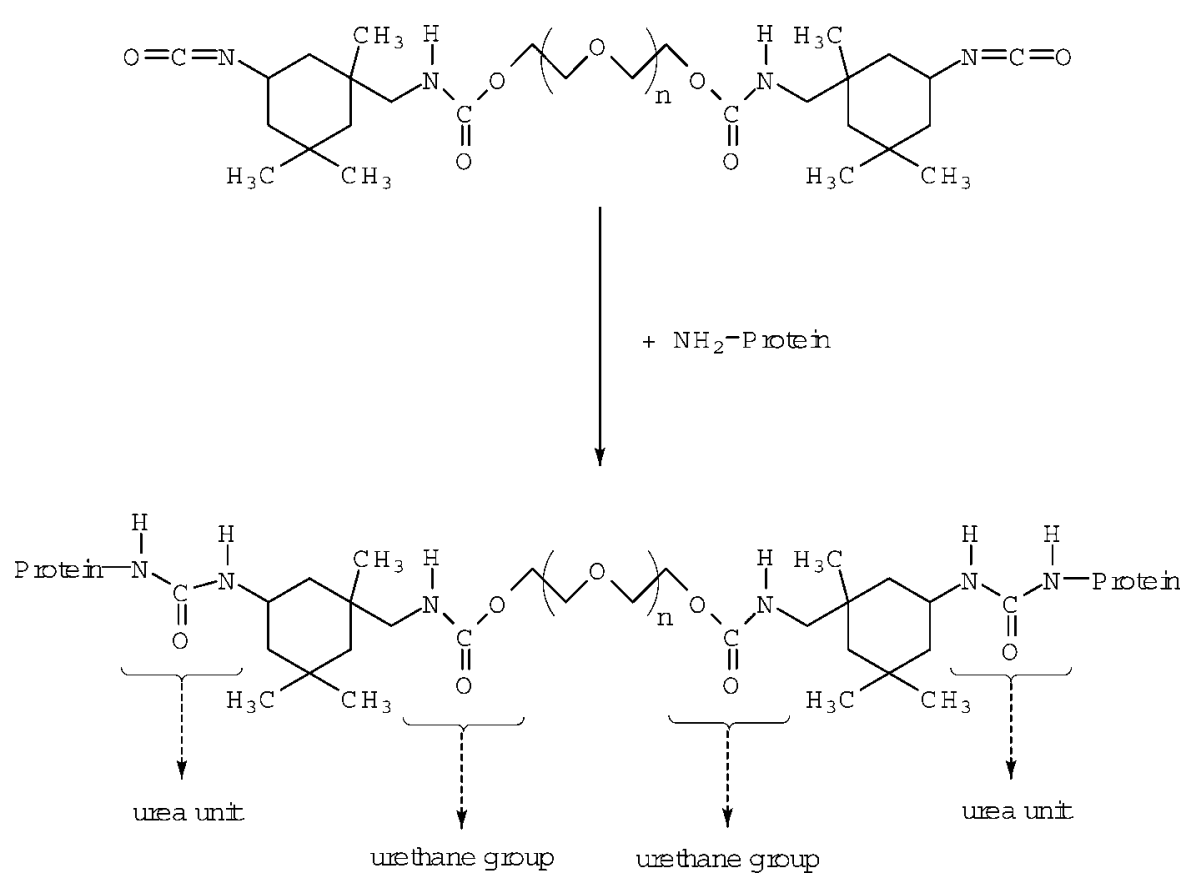

Figure 1 Reaction between a prepolymer and the amino groups of a protein resulting in a urea linkage.

the constrained layer damping mode, with a standard heating rate of $2^{\circ} \mathrm{C} / \mathrm{min}$, in a multifrequency mode $(1,3$, and $10 \mathrm{~Hz})$, and with a displacement of 0.05 $\mathrm{mm}$. The glass-transition temperature $\left(T_{g}\right)$ was determined as the peak in $\tan \delta\left(\tan \delta=E^{\prime \prime} / E^{\prime}\right.$, where $E^{\prime \prime}$ and $E^{\prime}$ are the loss and storage moduli, respectively, derived from DMTA). The differential scanning calorimetry (DSC) curves were also obtained at a heating rate of $2^{\circ} \mathrm{C} / \mathrm{min}$ in a TA Q100 from Thermal Analysis for a sample with a mass of $6 \mathrm{mg}$. The thermogravimetric analysis (TGA) was carried out in an SDT Q600 from Thermal Analysis, again at a $2^{\circ} \mathrm{C} / \mathrm{min}$ heating rate. A sample with a mass of $11 \mathrm{mg}$ was used to perform this analysis. Both DSC and TGA were performed in a nitrogen atmosphere at a flow rate of $100 \mathrm{~mL} / \mathrm{min}$.

\section{Hemocompatibility}

The hemocompatibility was evaluated in vitro according to International Standard Organization Standard $10993-4{ }^{16}$ and two different types of blood interactions were studied: thrombogenicity and hemolysis.

\section{Thrombogenicity}

The evaluation of thrombus formation on the polymeric surfaces was carried out with the gravimetric method of Imai and Nose. ${ }^{17}$ Anticoagulated rabbit blood was used for this purpose. This sample was prepared by the addition of $1 \mathrm{~mL}$ of an ACD solution to $9 \mathrm{~mL}$ of fresh rabbit blood. Before the tests, the urethanes were incubated with phosphate buffered sa- line (PBS; $\mathrm{pH} 7.4$ ) at a constant temperature of $37^{\circ} \mathrm{C}$. After $24 \mathrm{~h}$ of incubation, the PBS was removed, and the ACD blood was added to the polymers and also to an empty Petri dish, which acted as a positive control. The blood-clotting tests were initiated by the addition of $0.02 \mathrm{~mL}$ of a $0.10 \mathrm{M}$ calcium chloride solution and were stopped by the addition of $5 \mathrm{~mL}$ of water after $45 \mathrm{~min}$. The resultant clots were fixed with $5 \mathrm{~mL}$ of a formaldehyde solution $(36 \%)$, then dried with tissue paper, and finally weighed.

\section{Hemolysis}

The hemolysis tests were performed as described in American Society for Testing and Materials (ASTM) Standard F 756-00. ${ }^{18}$

Three samples of each urethane $\left(21 \mathrm{~cm}^{2}\right)$ were placed in polypropylene test tubes, and $7 \mathrm{~mL}$ of PBS was added. After $72 \mathrm{~h}$ of incubation at $37^{\circ} \mathrm{C}$, the PBS was removed, and $1 \mathrm{~mL}$ of diluted ACD venous rabbit blood $(9.02 \mathrm{mg} / \mathrm{mL})$ was added to each sample. ACD blood was also added both to the PBS extraction solution and to three samples of prepolymers with no previous treatment with PBS, and they were all maintained at $37^{\circ} \mathrm{C}$ for $3 \mathrm{~h}$. Positive and negative controls were prepared by the addition of the same amount of ACD blood to $7 \mathrm{~mL}$ of water and PBS, respectively. Each tube was gently inverted twice each $30 \mathrm{~min}$ to maintain the contact of the blood with the material. After incubation, each fluid was transferred to a suitable tube and centrifuged at $2000 \mathrm{rpm}$ for $15 \mathrm{~min}$. The hemoglobin released by hemolysis was measured by the optical densities (ODs) of the supernatants at 


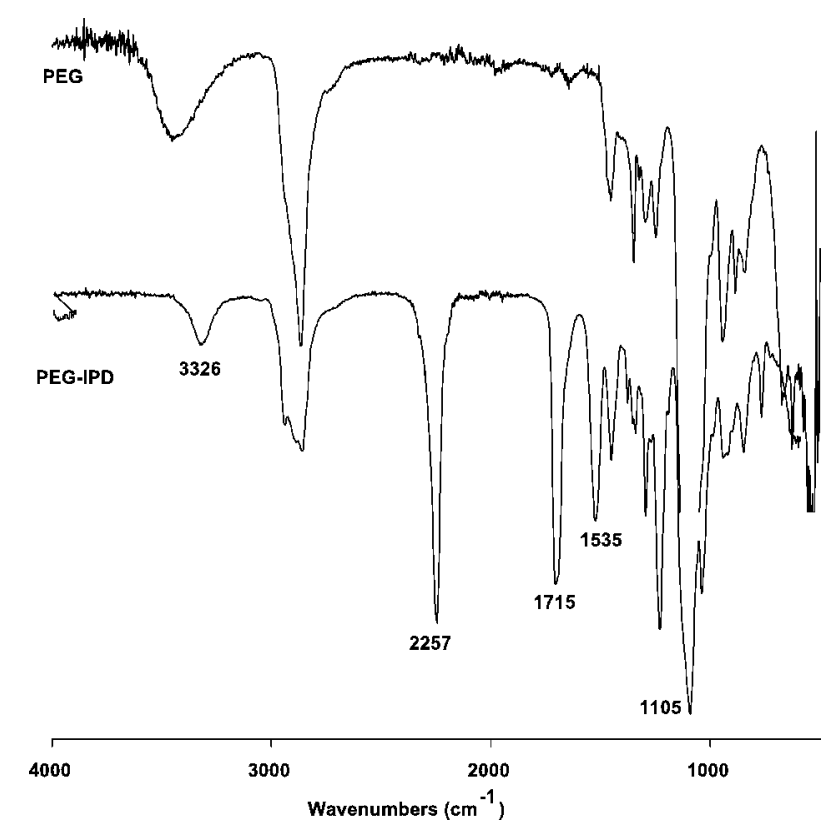

Figure 2 ATR-FTIR spectra obtained for PEG 400 and PEG-IPD urethane.

$540 \mathrm{~nm}$ with an ultraviolet-visible spectrophotometer (V-550, Jasco). The percentage of hemolysis was calculated as described in eq. (2):

$\%$ Haemolysis $=\left(\frac{O D_{\text {test }}-O D_{\text {negative control }}}{O D_{\text {positive control }}-O D_{\text {negative control }}}\right)$

\section{RESULTS AND DISCUSSION}

\section{Synthesis}

A prepolymer with free isocyanate groups was synthesized by the reaction of PEG 400 with IPD. The urethane was prepared in a 2/1 ratio of NCO groups to $\mathrm{OH}$ groups to obtain a polymer with terminal isocyanate groups.

The formation of the urethane groups was monitored by ATR-FTIR by the detection of the bands at $1715 \mathrm{~cm}^{-1}$ (free $\mathrm{C}=\mathrm{O}$ stretching in urethane) and at $1535 \mathrm{~cm}^{-1}(\mathrm{C}-\mathrm{N}$ stretching and $\mathrm{N}-\mathrm{H}$ bending from the urethane group; Fig. 2). It was also observed that all PEG hydroxyl groups reacted with the NCO groups of the isocyanate because the band that corresponded to the $\mathrm{OH}$ groups $\left(3452 \mathrm{~cm}^{-1}\right)$ was no longer detected in the urethane spectra. Instead, a new band was detected at $3326 \mathrm{~cm}^{-1}$ corresponding to $\mathrm{N}-\mathrm{H}$ hydrogen-bonded stretching. The presence of $\mathrm{NCO}$

$$
-\mathrm{NCO}+\mathrm{H}_{2} \mathrm{O} \longrightarrow-\mathrm{NH}_{2}+\mathrm{CO}_{2}
$$

Figure 3 Reaction between an isocyanate and water.

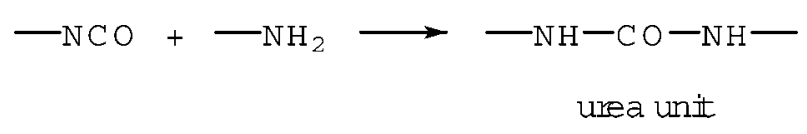

Figure 4 Reaction between isocyanate and aminic groups with the formation of a urea unit.

free groups on the prepolymers was also proved by the same technique because one could detect the free NCO stretching band around $2257 \mathrm{~cm}^{-1}$. At 1105 $\mathrm{cm}^{-1}$ was located the band corresponding to the $\mathrm{C}-\mathrm{O}-\mathrm{C}$ stretching in the aliphatic polyether, that is, in PEG.

\section{Swelling-capacity evaluation and moisture-curing kinetics}

The value obtained for the swelling ratio of the urethane was $19.7 \%$, which means that the prepolymer can be classified as an hydrogel.

The swelling capacity of a polymer is a very important parameter when we are dealing with biological applications. If a polymer swells when in contact with living tissues, this may mean that it will damage the surrounding tissues because of the increase in its volume. This phenomenon can be the main factor responsible for a deficient healing process and possible secondary effects such as infections and inefficient cicatrisation.

Considering the results obtained when we evaluated this parameter, we can suggest that the urethanes will not increase their volume in a matter that can prevent their use for the intended purpose. However, they still present some hydrophilicity, which can contribute to their biocompatibility.

One of the advantages of the urethanes is their ability to promote adhesion by reacting with the amino groups of the proteins existing in the living tissues; the importance of analyzing the stability of NCO free groups becomes evident, even under dramatic conditions such as the ones represented by a water-saturated atmosphere.

The isocyanate end groups present in this type of polymer are able to react with air moisture, resulting in the formation of carbon dioxide and an aminic ending polymer, ${ }^{19}$ as presented in Figure 3.

However, further reactions may occur, resulting first in the formation of urea groups (Fig. 4). Finally, the isocyanate groups may react with these amino units, leading to a crosslinked network (Fig. 5). ${ }^{19}$

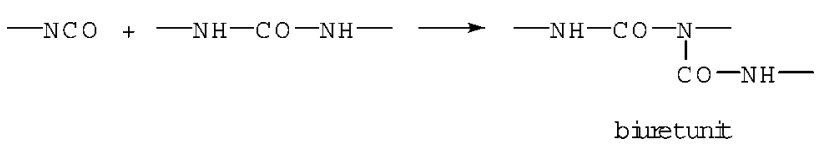

Figure 5 Reaction between isocyanate and urea units resulting in the crosslinking of the urethane. 


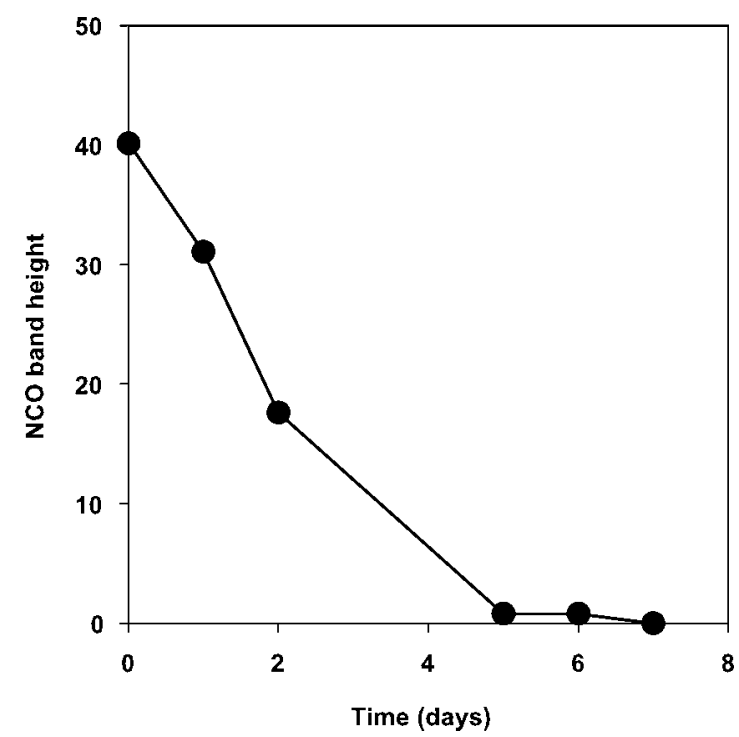

Figure 6 Evolution of the band height corresponding to the NCO free groups.

Although this moisture curing of the adhesive will necessarily occur in the living tissues, it is also important to avoid this occurrence while the adhesive is still not being used, such as when it is being manipulated or even is in storage.

The stability of the NCO groups, in the presence of some humidity, was evaluated by the placement of the samples in a water-saturated atmosphere and by the monitoring of the evolution of the height of the band at $2257 \mathrm{~cm}^{-1}$ by the ATR-FTIR technique, which corresponds to the NCO free group stretching.

The results shown in Figure 6 suggest that after 7 days all the NCO groups were hydrolyzed and half of them were hydrolyzed after 2 days of moisture exposure. Considering, however, that the tests were performed in an atmosphere saturated in water, we think that the NCO groups seem to be stable enough to be kept under storage conditions as long as humidity is avoided until its application is intended and also during the surgeon's manipulation.

When we consider the application conditions, it is important to keep in mind that water will be present in the living tissues. However, it is well known that the reactivity of isocyanate with water and primary hydroxyl groups is much less than that with amines. In fact, if we consider the relative reaction rate of the isocyanate group with water without a catalyst to be 100 at $25^{\circ} \mathrm{C}$, the same reaction would present a rate of 100,000 with a primary aliphatic amine and a rate of 20,000-50,000 with a secondary aliphatic amine. ${ }^{20}$

Taking these effects in consideration, we expect the reaction between NCO and amino groups in the living tissues to occur much faster than the one between $\mathrm{NCO}$ and water. However, it may be unavoidable that some does happen.

\section{Reaction with aminated substrates}

As already reported in the literature, ${ }^{20}$ the reaction of isocyanate groups with amines, forming urea linkages, is very fast and does not require catalysis.

The binding capacity of the adhesive was determined by its introduction between two pieces of gelatin that were either dry or moisturized with plasma. These were then subjected to the binding strength test, which terminated either with the fracture of the gelatin sheets or their separation if adhesion failed to occur. The values of the maximum force in the curve were registered and are presented in Figure 7.

It was observed that the urethane samples were able to bind the two gelatin sheets together, under either dry or moisture conditions. There was, however, a decrease in the peak force when humidity was present. This can be explained by the weakness of the gelatin structure caused by the plasma rather then by a decrease in the efficiency of the adhesive because the gelatin remained glued together.

\section{Determination of the surface energy by contact-angle measurements}

The main purpose of this measurement was to evaluate if the adhesive would spread when placed over an aminated substrate, over skin, and over a bleeding surface. Skin has a critical surface energy that varies between 38 and $56 \mathrm{mN} / \mathrm{m}$, depending on its temperature and relative humidity. ${ }^{21}$ The surface tension of blood, assessed for a group of 150 healthy people (72 men and 78 women ranging from 20 to 65 years old) by the drop-weight method at a temperature of $22^{\circ} \mathrm{C}$,

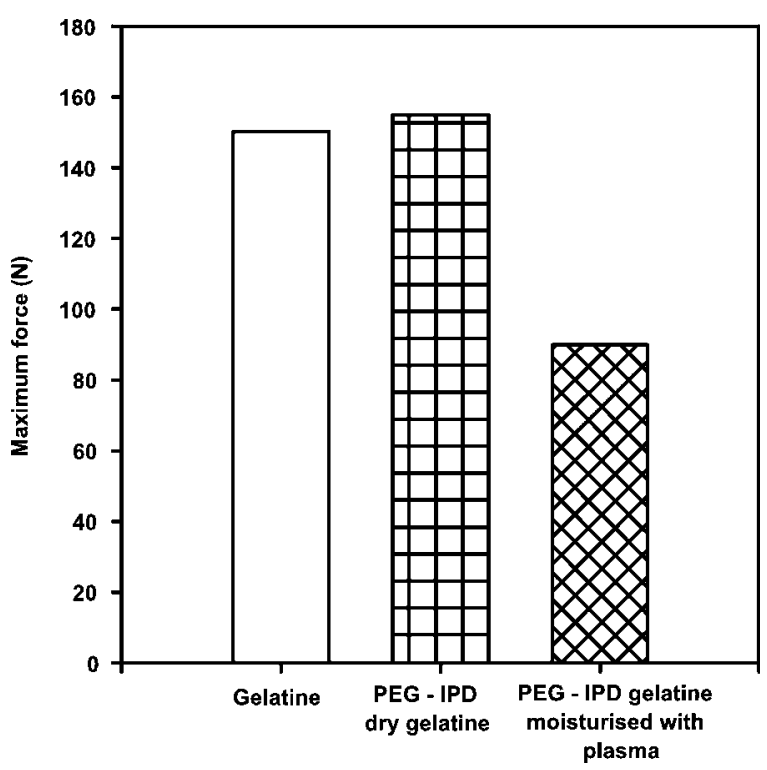

Figure 7 Values registered for the maximum force during binding strength tests for the urethane under dry and moisturized conditions. 
TABLE I

Surface Energy and Surface Tension Values and Corresponding Dispersive and Polar Components

\begin{tabular}{lccc}
\hline Substrate & $\gamma_{s}(\mathrm{mN} / \mathrm{m})^{\mathrm{c}}$ & $\gamma_{s}^{d}(\mathrm{mN} / \mathrm{m})^{\mathrm{d}}$ & $\gamma_{s}^{p}(\mathrm{mN} / \mathrm{m})^{\mathrm{e}}$ \\
\hline Gelatin & 44.24 & 5.00 & 39.24 \\
Skin $^{\mathrm{a}}$ & $38-56$ & - & - \\
Blood $^{\mathrm{b}}$ & 63.80 & - & - \\
PEG-IPD & 14.87 & 9.35 & 5.52 \\
\hline
\end{tabular}

a The data were taken from ref. 21.

$\mathrm{b}$ The data were taken from ref. 22 .

c Surface tension.

${ }^{\mathrm{d}}$ Dispersive component.

e Polar component.

was $63.8 \mathrm{mN} / \mathrm{m} .{ }^{22}$ Considering these parameters, we find that it is important to compare them with the values obtained for synthesized materials. In fact, for any adhesive to adhere to a substrate, one fundamental thermodynamic requirement has to be satisfied: the measured surface energy of the adhesive must be equal to or less than that of the adherent.

To evaluate this parameter, the surface tensions of the prepolymer and gelatin sheets were evaluated, by static contact-angle measurements, with four liquids: water, formamide, ethylene glycol, and propylene glycol. According to the Owens-Wendt-Rabel and Kaelble relationships, the dispersive and polar components of the urethane and the gelatin sheets were determined. The obtained results are presented in Table I.

The obtained results showed that the surface energies of gelatin, skin, and also blood are much higher than that of the prepolymer. This means that the adhesive forces between any of these surfaces and the urethane will overcome the cohesive forces of the prepolymer, and intermolecular proximity between the adhesive and the surface will, for this reason, occur. Consequently, adhesion between the urethane and either skin or blood is likely to happen.

\section{Thermal properties}

If we intend to apply a polymer as a surgical adhesive, its viscoelastic and thermal properties have to be evaluated. DMTA has been intensely used and is recognized as a powerful tool for identifying the thermal transitions of materials because of its extremely high sensitivity. Its special sensitivity for determining frequency-dependent transitions, such as $T_{g}$, which represents the motions of long-chain segments, is of outstanding importance. The basic function of DMTA is to obtain information about the mechanical and thermal properties of materials by the application of a sinusoidal load to a specimen and the measurement of the resultant deformation while the sample is subjected to a controlled temperature program. The load depends on the material and sample geometry but normally is set at very low values, which make it possible to detect small changes in the mechanical and thermal behavior of the material.

DMTA was carried out in the constrained layer damping mode, which was previously used by the authors for different materials with high levels of success. $^{23-26}$ PEG 400 is a liquid material in a molten state at room temperature and is widely used in the preparation of copolymers. This polymer has a $T_{g}$ around $-70^{\circ} \mathrm{C}$ and a melting point around $6^{\circ} \mathrm{C}$ (Melting enthalpy $\left.\left(\Delta H_{m}\right)=118.4 \mathrm{~J} / \mathrm{g}\right){ }^{27,28}$ The DMTA trace of the urethane based on PEG 400 is presented in Figure 8.

The trace shows that the addition of the urethane groups to PEG 400 led to the shift of the $T_{g}$ to higher values $\left(-13^{\circ} \mathrm{C}\right)$ and naturally the increase in the $T_{g}$ values with the frequency used. These results follow the same trend described by Hiltunen et al. ${ }^{29}$ The authors synthesized polyurethanes based on lactic acid by modifying this molecule with IPD. They concluded that the stiff structure of the diisocyanate caused a significant enhancement of the final $T_{g}$ of the modified polymer.

The sensitivity of this equipment to these types of transitions ( $\alpha$ relaxations) allows us to distinguish without doubt the $T_{g}$ from the processes that are not frequency-dependent, such as crystallization $\left(78^{\circ} \mathrm{C}\right)$, at which the value remains constant, regardless of the frequency considered. The apparent activation energy $(\Delta H)$ for the $\alpha$ relaxation can be determined from the Arrhenius rate relationship:

$$
\ln f=-(\Delta H / R T)
$$

where $f$ is the frequency of analysis, $R$ is the gas constant, and $T$ is the peak temperature $(\mathrm{K})$. The $\Delta H$ value obtained for this new material is $108.0 \mathrm{~kJ} / \mathrm{mol}$. It was also possible to observe a crystallization transi-

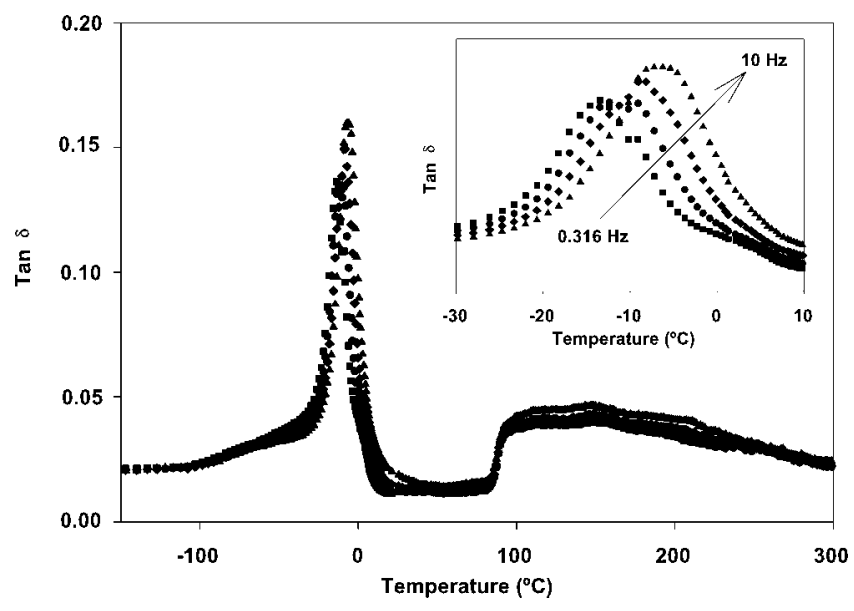

Figure 8 Multifrequency $\tan \delta$ trace obtained for PEGIPD. 


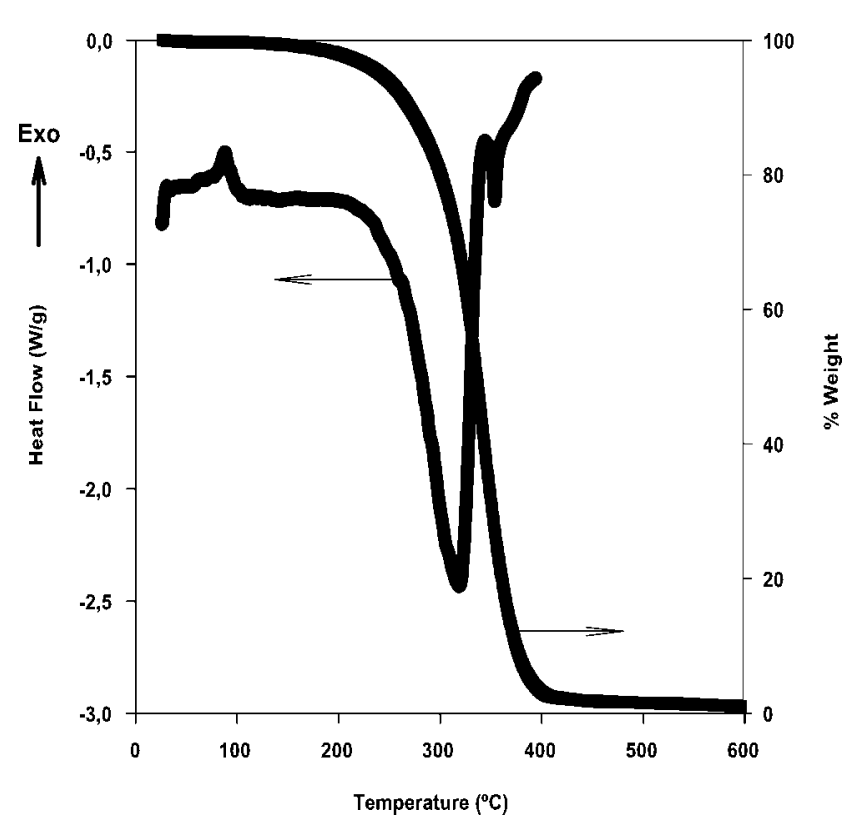

Figure 9 Results of DSC and TGA obtained for PEG-IPD at a heating rate of $2^{\circ} \mathrm{C} / \mathrm{min}$.

tion at the temperature of $84^{\circ} \mathrm{C}$ in the DMTA trace, which was also confirmed by an analysis of the DSC trace (Fig. 9), which shows an exothermic transition $\left(88^{\circ} \mathrm{C}\right)$ in the same region (crystallization enthalpy $\left.\left(\Delta H_{c}\right)=3.49 \mathrm{~J} / \mathrm{g}\right)$. These results show that PEG 400 was successfully modified, leading to a semicrystalline material. In addition, the DSC trace presents an extensive degradation transition with onset at $200^{\circ} \mathrm{C}$. The same value can be observed and therefore confirmed in the thermogravimetric curve (Fig. 9).

\section{Hemocompatibility}

Thrombogenicity

The capacity for thrombus formation of the urethane was evaluated by a gravimetric method. The medium weights of the blood clots formed after $45 \mathrm{~min}$ in three similar samples are presented in Figure 10.

It was observed that the clot formation was rather high for the urethane, even higher than that of the control. Considering that glass, the positive control, is considered a highly thrombogenic material, we can conclude that our material can also be classified as thrombogenic. This characteristic is directly related to the low surface energy presented by the urethane. The surface energy can be understood as a measure of the hydrophobicity/hydrophilicity. A high surface energy means that the surface is hydrophilic, and a low surface energy characterizes the surfaces as hydrophobic. In low-surface-energy materials, proteins adsorb strongly and irreversibly, and in highsurface-energy materials, proteins adsorb weakly and reversibly. Once the protein adhesion constitutes the first step to initiate the coagulation cascade, which ends in thrombus formation, it is expected that the urethane will present a high thrombogenic character. For the majority of the biomaterials, especially the ones designed to act as implants, this would be an undesirable feature, but because the adhesives would be applied under blooding conditions, its hemostatic character is of great importance. This material would largely contribute to stopping bleeding, initiating coagulation, and therefore helping the cicatrisation of the wound.

An interesting point is the fact that usually PEG is used to modify surfaces to diminish the thrombus formation. However, some points have to be taken into consideration. When PEG is used to modify surfaces, it is usually covalently coupled to the surfaces, the remaining structure being kept intact. Jeon and Andrade $^{30}$ treated the protein resistance of grafted PEG chains. According to the authors, the resistance of the surfaces treated with PEG to protein adsorption was related to its high hydrophilicity. In the mechanism proposed by them, as the protein approaches the surface, the water molecules associated with the hydrated PEG chains are compressed out of the PEG layer. However, this water removal is thermodynamically unfavorable and leads to steric repulsion, resulting in the inertness of the surfaces with covalently linked PEG. The authors also stated that this inertness increases with the length and density of the PEG chains. This means that shorter chains of PEG will present lower resistance to protein adsorption. Also, in our study, the PEG molecules were modified in their hydroxyl groups, which corresponded to diminished swelling. In fact, its maximum in swelling was lower than $20 \%$, and probably for that reason, the interactions with water molecules were less significant, and thrombosis occurred.

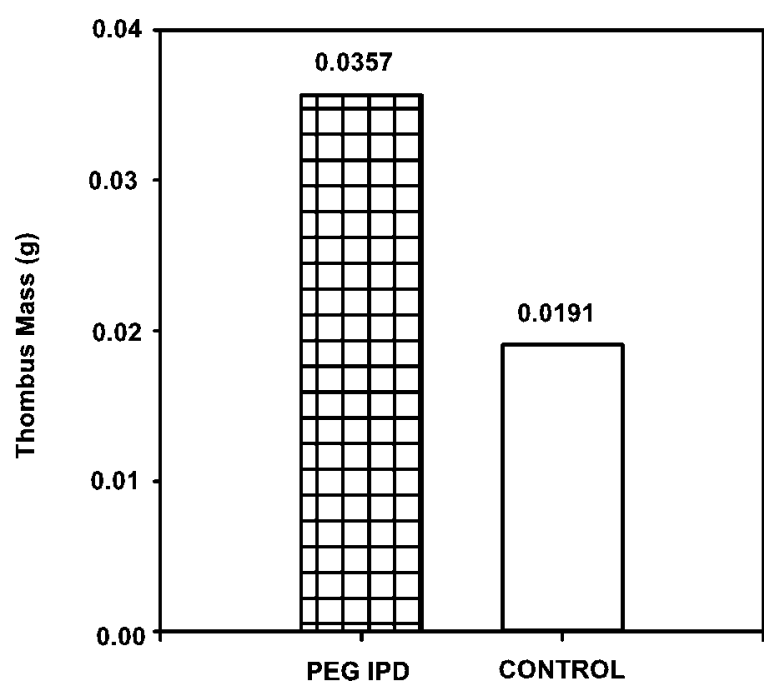

Figure 10 Weight of the thrombus formed after $45 \mathrm{~min}$. 


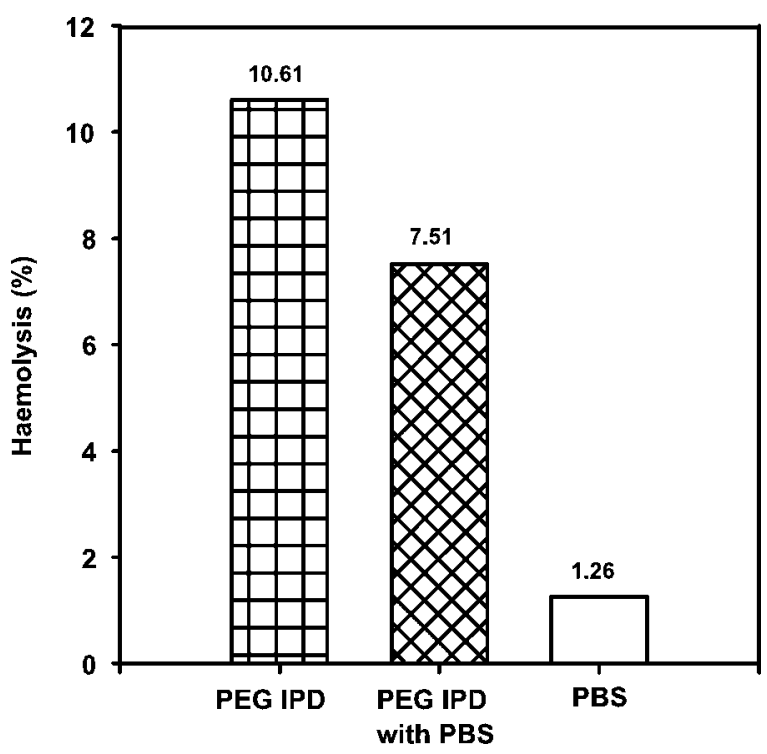

Figure 11 Values of the hemolysis of the urethanes and PBS in contact with them.

\section{Hemolysis}

During a hemolysis assay, the test and control materials were placed in contact with ACD rabbit blood under identical conditions, and the increase in the released hemoglobin was measured.

The values of hemolysis were obtained for samples in direct contact with blood, samples that were kept with PBS (indirect contact), and the PBS extraction solution (Fig. 11).

According to ASTM F 756-00, ${ }^{18}$ materials can be classified into several categories according to their hemolytic index. When this value is between 0 and $2 \%$, the material is classified as nonhemolytic. If hemolysis reaches values between 2 and 5\%, the material is classified as slightly hemolytic. However, when materials induce values of hemolysis superior to $10 \%$, they are classified as hemolytic.

The synthesized urethane can be classified as hemolytic because its hemolysis percentage is slightly above $10 \%$. This value, however, decreased to around $7.5 \%$ when it was incubated with the saline solution, and this indicates that part of the hemolysis is caused by products that can be washed away by the PBS, probably monomers that do not react during the synthesis.

Hemolysis is regarded as an especially significant screening test, once it provides quantification of small levels of plasma hemoglobin, which may not be measurable under in vivo conditions. According to ASTM Standard F $756-00,{ }^{18}$ it is not possible to define a universal level of acceptable or unacceptable amounts of hemolysis. Although by definition a blood-compatible material should be nonhemolytic, the truth is that in practice several medical devices cause hemolysis. This means that when such a hemolytic effect takes place, it is important to make sure that clinical benefits overcome the risks and that the values of hemolysis are within acceptable limits. It is also important to emphasize the fact that the intended application for this material does not imply its contact with blood flow for a large amount of time, and for that reason, we do not consider $10 \%$ hemolysis a prohibiting value.

\section{CONCLUSIONS}

A urethane was synthesized by the reaction of PEG 400 with IPD. The polymer was prepared in a 2/1 ratio of $\mathrm{NCO}$ to $\mathrm{OH}$ to end up with terminal isocyanate groups. The occurrence of the synthesis reaction was confirmed by both ATR-FTIR and DMTA. The prepolymer did not exhibit a high swelling ratio, so the effect of damaging the surrounding tissues due to the increase in its volume would not be considerable. Also, the NCO groups were found to be stable enough to be kept under storage conditions as long as humidity was avoided. Even under water-saturated conditions, it took 7 days for the hydrolysis of all the NCO groups to occur. The surface energy of the urethane was proved to be low, and this was consistent with the high thrombosis value obtained. Because the synthesized material will be applied under bleeding conditions, its haemostatic potential will constitute an advantage. When hemolysis tests were performed, it was concluded that the urethane presented a hemolytic character. This result does not mean, however, that this material cannot have a clinical application.

The authors thank the Serviço de Imunohemoterapia at the Hospital da Universidade de Coimbra for supplying the human plasma.

\section{References}

1. Sheikh, N.; Katbab, A. A.; Mirzadeh, H. Int J Adhes Adhes 2000, 20, 299.

2. Dunn, C. J.; Goa, K. L. Drugs 1999, 58, 863.

3. Lerner, R.; Binur, N. S. J Surg Res 1990, 48, 165.

4. Silver, F. H.; Wang, M.; Pins, G. D. Biomaterials 1995, 16, 891.

5. (a) King, M. E.; Kinney, A. Y. Nurse Pract Oct 1999, 24, 66; (b) King, M. E.; Kinney, A. Y. Nurse Pract Oct 1999, 24, 69; (c) King, M. E.; Kinney, A. Y. Nurse Pract Oct 1999, 24, 73.

6. Hida, T.; Sheta, S. M.; Proia, A. D.; McCuen, B. W. Retina $1988,8,148$.

7. Leahey, A. B.; Gottsch, J. D.; Stark, W. J. Ophthalmology 1993, 100, 173.

8. Donkerwolcke, M.; Burny, F.; Muster, D. Biomaterials 1998, 19, 1461.

9. Matsuda, S.; Iwata, H.; Se, N.; Ikada, Y. J Biomed Mater Res 1999, 45, 20.

10. Bingley, J. A.; Gardner, M. A. H.; Stafford, E. G.; Mau, T. K.; Pohlner, P. G.; Tam, R. K. W.; Jalali, H.; Tesar, P. J.; O'Brien, M. F. Ann Thorac Surg 2000, 69, 1764.

11. Lipatova, T. E. Adv Polym Sci 1986, 79, 65. 
12. Nosov, A. T.; Poliakov, A. A. Klin Khir 1980, 12, 16.

13. Komissarenko, I. V.; Kebuladze, I. M.; Lysenko, A. G.; Shumova, T. V. Klin Khir 1985, 12, 19.

14. Zemskov, V. S.; Biletskii, V. I.; Panchenko, S. N.; Shchitov, V. S.; Blagodarov, V. N. Klin Khir 1986, 11, 3.

15. ACD-Anticoagulant Citrate Dextrose Solution; United States Pharmacopeia: 1995; Vol. 23, p 119.

16. Biological Evaluation of Medical Devices-Part 4: Selection of Tests for Interaction with Blood; ISO 10990-4; International Standard Organization: Geneva, Switzerland, 1999.

17. Imai, Y.; Nose, Y. J Biomed Mater Res 1972, 6, 165.

18. Standard Practices for Assessment of Haemolytic Properties of Materials; ASTM F 756-00; American Society for Testing and Materials: West Conshohocken, PA, 2000.

19. Comyn, J.; Brady, F.; Dust, R. A.; Graham, M.; Haward, A. Int J Adhes Adhes 1998, 18, 51.

20. Vilar, W. D. Chemistry and Technology of Polyurethanes; Vilar Consultoria: Rio de Janeiro, Brazil, 2004; Chapter 1.2.

21. Venkatraman, R. G. Biomaterials 1998, 19, 1119.
22. Kratochvíl, A.; Hrnčíř, E. Physiol Res 2001, 50, 433.

23. Santos, K. S. C. R.; Coelho, J. F. J.; Ferreira, P.; Pinto, I.; Lorenzetti, S. G.; Ferreira, E. I.; Higa, O. Z.; Gil, M. H. Int J Pharm 2006, 310, 37.

24. Ferreira, P.; Coelho, J. F. J.; dos Santos, K. S. C. R.; Ferreira, E. I.; Gil, M. H. J Carbohydr Chem 2006, 25, 233.

25. Coelho, J. F. J.; Silva, A. M. F. P.; Popov, A. V.; Percec, V.; Abreu, M. V.; Gonçalves, P. M. O. F.; Gil, M. H. J Polym Sci Part A: Polym Chem 2006, 44, 2809.

26. Coelho, J. F. J.; Silva, A. M. F. P.; Popov, A. V.; Percec, V.; Abreu, M. V.; Gonçalves, P. M. O. F.; Gil, M. H. J Polym Sci Part A: Polym Chem 2006, 44, 3001.

27. Feldstein, M. M.; Shandryuk, G. A.; Kuptsov, S. A.; Platé, N. A. Polymer 2000, 41, 5327.

28. Feldstein, M. M.; Shandryuk, G. A.; Kuptsov, S. A. Polymer 2000, 41, 5339.

29. Hiltunen, K.; Seppälä, J. V.; Härkönen, M. J Appl Polym Sci $1996,64,865$

30. Jeon, S. I.; Andrade, J. D. J Colloid Interface Sci 1991, 142, 159. 